\title{
Les trois fautes de goût de Quintus Arrius (Catulle 84)
}

Résumé : La lecture que nous proposons du carmen 84 de Catulle a pour but d'explorer certaines questions esthétiques liées à l'elocutio qui se posaient avec acuité aux auteurs du $\mathrm{I}^{\mathrm{er}}$ siècle avant notre ère. Il s'agissait là de points délicats à la fois pour les poètes et pour les orateurs, dont l'examen permet d'apporter une contribution à l'étude des rapports entre prose et poésie à la fin de l'époque républicaine. Nous reprenons, dans cette perspective, le dossier déjà épais concernant la prononciation intempestive d'aspirées reprochée à Arrius et celui, plus mince, qui porte sur un défaut d'élocution des sifflantes. Nous y ajoutons une proposition au sujet des hiatus qui suppose une interprétation de la pointe de l'épigramme différente des interprétations qui ont été avancées jusqu'à présent.

Abstract : The aim of this paper on Catullus 84 is to cast some light on aesthetic questions related to elocutio which had prime importance for $I^{\text {st }}$ century B.C. authors. These were thorny questions for both poets and orators and studying them helps understanding the links between prose and poetry in the late republican period. We reconsider, from this point of view, the well-known matter of Arrius' diction of the aspirates and the not so well-known matter of his mispronunciation of hissing sounds. We also suggest, by reading in a new way the point of the epigram, that Arrius may have had trouble with hiatus as well.

Chommoda dicebat, si quando commoda uellet

Dicere, et insidias Arrius hinsidias,

Et tum mirifice sperabat se esse locutum,

Cum quantum poterat dixerat hinsidias.

Credo, sic mater, sic liber aunnculus eius,

Sic maternus auus dixerat atque auia.

Hoc misso in Syriam requierant omnibus aures ;

Audibant eadem haec leniter et leuiter,

Nec sibi postilla metuebant talia uerba,

Cum subito affertur nuntius horribilis,

Ionios fluctus, postquam illuc Arrius isset,

Iam non Ionios esse sed Hionios.

$\left(\right.$ Catulle, 84) ${ }^{1}$

\footnotetext{
1 «Havantages », disait Arrius, quand il voulait dire «avantages », et « hembûches » au lieu d'«embûches »; il se figurait avoir parlé merveilleusement, quand il avait dit de toute sa force « hembûches ». Ainsi disaient, je crois, sa mère et l'affranchi, frère de sa mère, son aïeul et son aïeule maternels. Quand il fut envoyé en Syrie, ce fut le repos pour toutes les oreilles; elles entendaient ces mêmes mots sous leur forme douce et légère et n'en redoutaient plus d'autres pour l'avenir, quand tout à coup arrive une nouvelle horrible : la mer Ionienne, depuis qu'Arrius est allé là-bas, ne s'appelait plus Ionienne, mais « Hionienne ». (trad. Lafaye - Viarre, 2002, CUF)
} 
L'Arrius - Quintus Arrius probablement, s'il faut l'identifier ${ }^{2}$ - du poème 84 de Catulle disait, c'est bien connu, "hinsidias » au lieu de "insidias » et «chommoda » pour «commoda ». Mais peut-être prononçait-il aussi les sifflantes, surtout en position finale, avec une insistance excessive et émaillait-il ses discours de trop nombreux hiatus. Si Catulle a stigmatisé ces défauts d'élocution et si sa pièce a rencontré un large succès - Quintilien la qualifiait de nobile epigramma (Inst. I, 5, 20) -, c'est que ces erreurs n'étaient pas propres à un individu isolé. Elles devaient non seulement concerner l'ensemble d'un groupe social, mais aussi intéresser de manière particulière les cercles lettrés de l'époque. Notre hypothèse est, d'une part, que ces défauts dans l'elocutio touchaient à la question de l'empreinte de la culture grecque à Rome, un problème qui ne laissait aucun Romain indifférent. Nous pensons, d'autre part, que les fautes de goût d'Arrius devaient avoir, pour les Romains cultivés, une saveur particulière du fait qu'elles ne singeaient pas seulement leur manière de s'exprimer dans leurs conversations familières mais qu'elles constituaient une caricature des choix esthétiques qu'ils étaient amenés à faire dans leurs œuvres aussi bien oratoires que poétiques.

\section{Aspirations intempestives}

L'épigramme de Catulle épingle une erreur d'élocution d'Arrius consistant en la prononciation exagérée d'aspirations (quantum poterat, 4) dans des mots qui normalement n'en comportent pas (commodas, insidias, Ionios) ${ }^{3}$. Mais l'interprétation de ces aspirations

\footnotetext{
${ }^{2}$ L'identité de ce personnage a été discutée, mais il semble bien qu'il s'agisse de Quintus Arrius, parvenu d'humble origine, piètre orateur (Cic., Brut. 242-243), candidat malheureux au consulat en 58 (Cic., Att. II, 5, 2 et II, 7, 3) et associé de Crassus (Cic., Brut. 242-243), qu'il a pu accompagner en Syrie lors de son proconsulat en 55. Cette identification avec Q. Arrius a été défendue de manière détaillée par B. A. Marshall et R. J. Baker, "The Aspirations of Q. Arrius », Historia, 24, 1975, p. 220-231 (voir p. 229-230 en particulier); elle est également considérée comme la plus plausible par W. Kroll, Catull, Stuttgart, 1959, p. 257, C. J. Fordyce, Catullus, A Commentary, Oxford, 1961, p. 375, K. Quinn, Catullus: The Poems, Londres, $1973^{2}\left(1970^{1}\right)$, p. 419, F. Della Corte, Catullo. Le poesie, Rome, 1977, p. 344-345, et est reprise, plus récemment, par D. F. S. Thomson, Catullus, edited with a textual and interpretative commentary, Toronto, 1997, p. 512, J. Nicholson, «Catullus 84: In Vino Veritas? ", Phoenix, 52, 1998, p. 299-304, et L. F. Coraluppi, « Il carme 84 di Catullo », Acme, 52, 1999, p. 153-171. Certains chercheurs distinguent deux Q. Arrius, l'un préteur en 63, l'autre en 73 : voir E. S. Gruen, The Last Generation of the Roman Republic, Berkeley, 1974, p. 74 et, pour une discussion de ce point de vue, Marshall et Baker, 1975. Pour le rapprochement de l'Arrius de Catulle avec C. Arrius, un voisin importun qualifié de perurbanus par Cicéron (Att. 2, 14, 2 et 2, 15, 3, lettres écrites de Formies en avril 59), voir E. S. Ramage, « Note on Catullus' Arrius », CPh, 54, 1959, p. $44-$ 45 .

${ }^{3}$ Nous devons à Politien l'état actuel du texte. Dans les manuscrits anciens, le deuxième distique apparaissait en cinquième position, peut-être à la suite d'une omission (qui pourrait être liée à la répétition de insidias à la fin des deux premiers distiques), d'un ajout marginal pour la réparer et d'une réinsertion au mauvais endroit. D'autre part, la notation graphique par $h$ des aspirations n'apparaissait pas dans la tradition manuscrite (on trouvait deux fois commoda au v. 1, insidias aux v. 2 et 4 et Ionios au dernier vers ; les trois manuscrits principaux portaient toutefois insidias he ou insidias hee à la fin du v. 2); elle a été introduite sur la base du passage suivant de Quintilien : Parcissime ea ueteres usi etiam in uocalibus, cum " aedos" "ircos »que dicebant. Diu deinde seruatum ne consonantibus adspirarent, ut in "Graccis》 et " triumpis 》. Erupit breui tempore nimius usus, ut "choronae », " chenturiones », " praechones » adhuc quibusdam <in> inscriptionibus maneant, qua de re Catulli
} 
intempestives a varié. On a pu penser à l'influence de parlers italiques voisins - l'étrusque ou le vénète ${ }^{4}-$, hypothèses suggérées par les vers 5-6 qui, pris au premier degré, indiqueraient qu'il s'agit d'une prononciation familiale transmise de génération en génération ${ }^{5}$. Plusieurs critiques ${ }^{6}$ comprennent toutefois ces deux vers comme ironiques : credo, mis ici en relief en début de vers, a, quand il se trouve en incise, un emploi ironique bien documenté, l'accumulation des termes de parenté soulignée par la répétition de sic a un caractère hyperbolique propre à signaler une attitude ironique du locuteur et il est bien possible que Catulle parodie ici un passage du De oratore de Cicéron (III, 45), ex quo sic locutum esse eius patrem iudico, sic maiores, dans lequel Laelia est louée pour la pureté de sa langue, qui doit refléter la manière de s'exprimer de ses ancêtres nobiles. Catulle soulignerait ainsi que la famille maternelle d'Arrius est d'origine humble, voire servile, et que ce n'est certainement pas de ses ancêtres qu'il a hérité cette prononciation pleine d'affectation, ce qui le dénonce en tant que parvenu.

L'hypothèse généralement retenue est celle d'un "hyperurbanisme ${ }^{7}$ lié à la mode hellénisante à Rome car, devenue débile en latin à l'époque historique ${ }^{8}$, la prononciation de l'aspiration a été restaurée dans les milieux cultivés au $\mathrm{I}^{\mathrm{er}} \mathrm{s}$. avant notre ère, vraisemblablement sous l'influence du grec, dont l'aura à Rome était de plus en plus

nobile epigramma est. (Quint. Inst. I, 5, 19-20), « Les anciens usaient très sobrement de l'aspiration, même devant les voyelles, puisqu'ils disaient aedus et ircus. Puis, on a longtemps veillé à ne pas aspirer les consonnes comme dans Gracci et triumpi. Pendant un court laps de temps, a surgi l'usage abusif <inverse $>$, si bien qu'on lit sur des inscriptions choronae, chenturiones, praechones, et il y a, sur ce sujet, une épigramme bien connue de Catulle. » (trad. Cousin - André, 1975, CUF). Les corrections faites par l'humaniste italien sont généralement acceptées; pour des vues partiellement divergentes, voir E. Harrison, "Catullus, LXXXIV », CR, 29, 1915, p. 198-199, qui accepte chommoda et Hionios mais propose de lire insidiash qui noterait une prononciation presque débile, entre [s] et [h], de la sifflante finale, et H. B. Rosén, « Arrius' Speech Again (Catullus 84) », Mn., 14, 1961, p. 224-232, qui accepte hinsidias et Hionios, mais propose pour le premier mot du poème une lecture cōmoda, avec substitution d'une séquence voyelle longue suivie d'une consonne simple à une séquence voyelle brève suivie d'une consonne géminée. Sur la tradition manuscrite de l'œuvre de Catulle, voir en dernier lieu M. Bonvicini, Il novus libellus di Catullo. Trasmissione del testo, problematicità della grafia e dell'interpunzione, Cesena, 2012.

${ }^{4}$ Ce sont les hypothèses avancées respectivement par A. J. Bell, « Note on Catullus, 84 », CR, 29, 1915, p. 137-139, et par Harrison, 1915, qui supposent que les aspirations étaient particulièrement nombreuses dans ces langues.

${ }^{5}$ Voir encore J. M. Trappes-Lomax, Catullus : A Textual Reappraisal, Swansea, 2007, p. 258-259, qui propose tout bonnement de supprimer ce distique.

${ }^{6}$ Ainsi Fordyce, 1961, p. 376, Thomson, 1997, p. 513, Coraluppi, 1999, p. 155-156.

${ }^{7}$ Appellation proposée par N. I. Herescu, «Autour de l'ironie de Catulle », Rivista Classica, 13-14, 1941-1942, p. 128-137.

${ }^{8}$ La disparition complète de $h$, dans la graphie d'un certain nombre de mots (par exemple débeō < *dé-habeō, avec contraction, diribeō < *dis-habeō « je distribue », avec rhotacisme, nēmō < *nehemō, avec contraction) témoigne de cette évolution.

Pour les différents points de phonétique historique abordés dans ce paragraphe, on pourra se reporter aux manuels suivants : M. Niedermann, Précis de phonétique historique du latin, Paris, $1968^{4}\left(1904^{1}\right)$, p. 84-86 et 99-100, G. Meiser, Historische Laut- und Formenlehre der lateinischen Sprache, Darmstadt, $2006^{2}\left(1998^{1}\right)$, p. 105, M. Weiss, Outlines of the Historical and Comparative Grammar of Latin, Ann Arbor, New York, 2009, p. 62-63, W. S. Allen, Vox Latina. A Guide to the Pronunciation of Classical Latin, Cambridge, $1978^{2}\left(1965^{1}\right)$, p. 26-27 et 43-45, ainsi qu'à F. Biville, Graphie et prononciation des mots grecs en latin, Louvain, 1987, p. 30-31. 
importante. On peut de même remarquer l'introduction, vers la même époque, des digrammes $p h$, th et $c h$ pour noter les consonnes aspirées du grec, précédemment transcrites en latin par $p$, $t$ et $c^{9}$. On sait par ailleurs que la conservation du $h$ dans la graphie donnait lieu à des hypercorrections dont les inscriptions nous conservent des traces (hire, heius). La restauration de l'aspiration à l'oral, propre à l'élite culturelle urbaine, ne pouvait qu'entraîner de semblables confusions - des «hyperurbanismes » - de la part des snobs désireux d'imiter cette manie mais ne jouissant pas d'une culture suffisante pour savoir quels mots latins commençaient réellement par un $h^{10}$. Dans la langue littéraire même, d'ailleurs, plusieurs flottements peuvent être observés, concernant notamment les termes (h)anser (qui a tendance à perdre son aspiration d'origine), (h)umor, (h)umidus et (h)umerus (qui, eux, en acquièrent une). Ainsi Arrius prononçait-il, d'après Catulle, hinsidias. Le cas de chommoda est connexe, quoique légèrement différent. Il est lié à la manie hellénisante d'introduire dans la langue latine, en dehors même des mots empruntés au grec, des consonnes aspirées. Ainsi les Gracques s'étaient-ils fait appeler Gracchi, ainsi commençait-on à prononcer et à écrire pulcher au lieu de pulcer, comme en témoigne Cicéron ${ }^{11}$.

Si les chercheurs qui ont commenté cette épigramme ont remarqué que le défaut de prononciation d'Arrius était lié à l'hellénophilie ambiante, ils n'ont peut-être pas assez insisté sur deux autres aspects, à savoir ses résonances par rapport aux débats contemporains concernant l'elocutio chez l'orateur d'une part et par rapport à l'esthétique des poètes néotériques d'autre part. La nouvelle prononciation de l' $h$ latin et des consonnes aspirées dans les mots empruntés au grec est, comme on peut s'y attendre, débattue par ces spécialistes de la parole que sont les orateurs : le bon orateur prononcera-t-il ou non ces aspirations ? Cicéron (Orat. 160) opte finalement pour une prononciation des consonnes aspirées, bien qu'il sache qu'elle n'est pas conforme à l'usage traditionnel. Il estime en effet qu'il faut suivre en cela le jugement des oreilles. Cette importance accordée par l'orateur à l'agrément des sonorités ${ }^{12}$ trouve un écho dans la formulation adoptée par Catulle aux vers 7-8 (requierant omnibus aures, / audibant eadem haec leuiter et leniter). La question des consonnes aspirées paraît avoir été également importante pour les poètes néotériques comme Catulle. Il semble que ce soient eux, en effet, qui introduisirent dans la poésie latine l'usage d'un certain nombre de

\footnotetext{
${ }^{9}$ Les graphies $p h$, th et $c h$ apparaissent dans les inscriptions de la seconde moitié du II ${ }^{\mathrm{e}}$ siècle avant notre ère et commencent à s'imposer dans les inscriptions officielles au siècle suivant ; voir Biville, 1987, p. 30-31.

${ }^{10}$ C'est ce que nous apprend la remarque de Nigidius Figulus, un contemporain, conservée par AuluGelle (XIII, 6, 3) : Rusticus fit sermo si aspires perperam («On s'exprime sans distinction si l'on marque les aspirations à tort », trad. pers.) - remarque dont il ne faut pas déduire que l'aspiration était conservée dans les dialectes ruraux : rusticus doit s'entendre ici comme "grossier», sens que prend d'ailleurs aussi en français l'adjectif « rustique ».

${ }^{11}$ Orat. 160 : Quin ego ipse, cum scirem ita maiores locutos esse ut nusquam nisi in uocali aspiratione uterentur, loquebar sic ut "pulcros, Cetegos, triumpos, Cartaginem » dicerem; aliquando, idque sero, conuicio aurium cum extorta mihi ueritas esset, usum loquendi populo concessi, scientiam mihi reseruaui. "Moi-même, sachant que nos ancêtres ne mettaient d'aspiration nulle part que dans les voyelles, je prononçais pulcros, Cetegos, triumpos, Cartaginem. Une fois que, plus tard, les reproches de mes oreilles m'eurent fait abandonner mon souci de la correction, j'ai concédé au peuple le droit de fixer l'usage, et j'ai gardé ma science pour moi. » (trad. Yon, 1964, CUF)

${ }^{12}$ Voir Cic., Orat. 37-38, 162, 168, 170, 177-178, De orat. III, 181 et 191-192.
} 
termes empruntés au grec avec leur prononciation proprement grecque (consonnes aspirées, $z$, $y)$, douce et exotique aux oreilles latines ${ }^{13}$.

Si Catulle prend Arrius pour cible, ce n'est pas seulement parce qu'il est un parvenu mal dégrossi, c'est aussi parce qu'il est, plus particulièrement, un mauvais orateur. Son défaut de prononciation est la perversion d'un raffinement récemment introduit dans le discours oratoire et dans la poésie, et c'est en cela surtout qu'il exaspère Catulle ${ }^{14}$.

\section{Sigmatisme à tout va}

Il y a une vingtaine d'années, E. Vandiver ${ }^{15}$ a relevé la présence, dans l'épigramme de Catulle qui nous occupe, de nombreuses allitérations en [s] (42 occurrences de la sifflante dentale en 12 vers $^{16}$ ), et a remarqué en outre que ce son apparaît de manière récurrente en fin de vers et à la fin de mots de quatre syllabes commençant par un $h$ (hinsidias, 2 et 4 , horribilis, 10, Hionios, 12). Un vers, seul, ne comporte pas la moindre sifflante : le vers 8, qui décrit le soulagement éprouvé par les Romains au départ d'Arrius. Ces différentes observations amènent $\mathrm{E}$. Vandiver à penser qu'un second défaut de prononciation concernant la sifflante est ainsi critiqué indirectement par Catulle, proposition généralement acceptée depuis lors ${ }^{17}$. L'interprétation de cet autre défaut de prononciation n'est toutefois pas claire. E. Vandiver le lie à l'excès d'aspiration, mais l'existence d'un lien entre une prononciation forte de $[\mathrm{h}]$ et une prononciation forte de la sifflante est en fait loin d'être évidente. Si on l'admettait, cela indiquerait du reste un défaut de prononciation d'origine pathologique et conduirait donc à abandonner l'hypothèse, généralement admise pour l'aspiration, de l'hellénomanie.

Il nous semble possible de proposer une autre interprétation de ce déferlement de sifflantes, plus en conformité avec ce qui a pu être établi à propos des aspirations. La prononciation du $s$ final en latin - et E. Vandiver a justement souligné l'apparition répétée de

\footnotetext{
${ }^{13}$ Voir à ce propos Quintilien (Inst. XII, 10, 27-28); ces questions sont évoquées par R. G. G. Coleman, "Poetic Diction, Poetic Discourse and the Poetic Register», dans J. N. Adams et R. Mayer (dir.), Aspects of the Language of Latin Poetry, Oxford, 1999, p. 21-93 (voir p. 46). La date de l'introduction des graphèmes $Y$ et $Z$ dans les textes littéraires est difficile à déterminer; elle se fait dans les inscriptions au $\mathrm{I}^{\mathrm{er}}$ siècle avant notre ère. Nous disposons toutefois des témoignages de Cicéron (Orat. 160) et de Marius Victorinus $(G L 6,8,11)$ qui indiquent que ces graphies ne se trouvaient ni chez Ennius, ni chez Accius. Voir à ce propos F. Biville, Les Emprunts du latin au grec. Approche phonétique, Louvain - Paris, I, Introduction et consonantisme, 1990, p. 99-101 et II, Vocalisme et conclusions, 1995 , p. 257-260.

${ }^{14}$ Catulle se livre, de manière similaire, à des parodies stylistiques dans d'autres poèmes, notamment à l'encontre de Gellius, dont il ne partage pas les conceptions esthétiques en matière de poésie, à travers l'emploi d'un vers holospondaïque et l'élision d'un $s$ final en 116, ou du mauvais orateur Sestius en imitant l'affectation hors de propos et le mélange de tons incongru caractéristiques de la froideur (frigus) de son style. Voir respectivement C. W. MacLeod, « Catullus 116 », CQ 23, 1973, p. 304-309 (p. 307) et E. S. DeAngeli, « A Literary Chill: Catullus 44 », $C W$ 62, 1969, p. 354-356.

${ }^{15}$ E. Vandiver, « Sound Patterns in Catullus $84 », C J, 85,1990$, p. 337-340.

${ }^{16}$ Vandiver, 1990, ne fournit pas d'autres chiffres à titre de comparaison. Vérifications faites, la concentration en [s] est effectivement plus élevée dans le carmen 84 que dans le reste des épigrammes (3,5 vs 2,7 par vers en moyenne).

${ }^{17}$ Thomson, 1997, Nicholson, 1998, Coraluppi, 1999.
} 
$s$ en finale - est une autre question âprement débattue au $\mathrm{I}^{\mathrm{er}} \mathrm{s}$. avant notre ère ${ }^{18}$. Le $s$ final latin avait en effet tendance à être débile après une voyelle brève (pas au point d'entraîner une élision comme c'est le cas avec $-m$ final, mais de sorte qu'il ne faisait pas position devant un mot commençant par une consonne dans l'ancienne poésie latine), et c'est au cours du II siècle av. J.-C. que sa prononciation a été restaurée. Cicéron discute de la manière dont l'orateur doit prononcer cette consonne finale juste après avoir évoqué la question des consonnes aspirées (Orat. 161) : les gens distingués prononcent désormais ce $s$ - et il est à nouveau fait référence à ce qui charme l'oreille. On sait par ailleurs que les néotériques sont les premiers à refuser de recourir à cette licence pour garder une syllabe finale brève ${ }^{19}$, refus qui peut être lié à l'association de ce procédé avec le style épique ${ }^{20}$. L'unique omission assurée du $s$ final chez Catulle $(116,8)$ est ainsi considérée comme parodique du style prisé par Gellius, incapable d'apprécier les choix poétiques de Catulle ${ }^{21}$. Il semble donc s'agir là encore d'une question de prononciation liée à l'esthétique néotérique et aux débats des orateurs sur l'elocutio.

Il est, en outre, fort probable que cette question soit également en rapport avec l'hellénophilie. La restauration du $s$ final en latin est sans doute due en partie à une nécessité morphologique, l's final étant la marque de nombreuses désinences casuelles, mais elle intervient, tout comme la restauration de l'aspiration, à une époque où l'influence du grec, qui a bien conservé ses sifflantes finales, se fait fortement sentir à Rome ${ }^{22}$. L'abandon par les néotériques de la licence poétique concernant l's final pourrait également être expliqué par l'influence de la poésie grecque dont ces poètes tirent une grande partie de leur inspiration et dans laquelle ce type de licence n'existe pas.

Il semble, par ailleurs, que la récurrence des sifflantes soit un trait marquant chez un certain nombre de poètes grecs : Euripide, en particulier, aurait eu un goût prononcé pour les allitérations en sifflantes (il est moqué pour cela par les poètes comiques et qualifié de philosigmatos par Eustathe ${ }^{23}$ ) et cette tendance aurait été partagée, bien qu'à un moindre degré, par la plupart des poètes grecs de l'époque classique ${ }^{24}$. En revanche, les auteurs

\footnotetext{
${ }^{18}$ Messalla prit également position dans ce débat, d'après ce que rapporte Quintilien (Inst. IX, 4, 37), peut-être dans l'ouvrage qu'il consacra, toujours d'après Quintilien (Inst. I, 7, 35), à la lettre $s$.

${ }^{19}$ Lucrèce, volontiers archaïsant, y a encore recours à 41 reprises au moins (voir C. Dubois, Lucrèce, poète dactylique, Strasbourg, 1935, p. 12-14).

${ }^{20}$ Voir à ce sujet la synthèse de Coleman, 1999, p. 33-34. Dans sa récente édition des poèmes de Catulle, Trappes-Lomax, 2007, p. 6-8, défend l'idée que Catulle avait couramment recours à cette licence, mais ses arguments ne nous paraissent pas solides : les scribes auraient pu corriger le vers 8 du carmen 116 aussi bien que d'autres vers (voir le compte rendu de D. J. Butterfield, "Catullus semilautus », $C R, 59,2009$, p. 117-120, en particulier p. 119) et, surtout, Trappes-Lomax ne tient aucun compte de la valeur parodique du vers en question.

${ }^{21}$ Il s'agit d'un écho d'Ennius (Ann. 95 Sk.). Pour une interprétation de l'élision de ce $s$ final comme une imitation de la manière de Gellius, voir MacLeod, 1973, p. 307.

${ }^{22} \mathrm{~L}$. Michel, Étude du son $\mathrm{s}$ en latin et en roman des origines aux langues romanes, de la phonétique au style, Paris-Montpellier, 1955, p. 99, et Coleman, 1999, p. 34. Pour la description de l'amuïssement du $s$ final par les grammairiens latins et les rapprochements faits par ces derniers avec la scansion homérique, voir C. Conduché, «Priscien, De metris Terentii, 2-3 et l'application à la langue grecque de catégories grammaticales latines $", R P h$, à paraître.

${ }^{23}$ Eust., Com. ad Il. 19.47 (van der Valk 4.283.7).

${ }^{24}$ Pour les données concernant les auteurs grecs fournies dans ce paragraphe, nous nous fondons sur les articles de D. L. Clayman, "Sigmatism in Greek Poetry », TAPhA, 117, 1987, p. 69-84, O. J. Todd, "Sense and Sound in Classical Poetry », CQ, 36, 1942, p. 29-39, J. A. Scott, "Sigmatism in Greek Dramatic Poetry», AJPh, 29, 1908, p. 69-77 et «The Effect of Sigmatism as Shown in Homer »,
} 
alexandrins comme Callimaque, dont s'inspirent plus particulièrement les néotériques, font preuve de beaucoup plus de circonspection en ce domaine. On peut donc penser que la question de l'euphonie, qui prescrivait, dans l'Antiquité grecque et latine, d'éviter l'excès de sifflantes ${ }^{25}$ entre aussi en jeu ici. La nature même de la cacophonie particulière que constitue le sigmatisme n'est toutefois pas clairement établie : s'agit-il simplement de la concentration d'un nombre élevé de sifflantes dans un même vers ou plutôt de la disposition des sifflantes à l'intérieur du vers? La plupart des études portant sur ce phénomène en latin et en grec s'intéressent au premier aspect et produisent des statistiques sur le nombre moyen de sifflantes par vers ou sur le nombre de vers présentant une concentration remarquable de sifflantes. Il en ressort que les vers les plus sigmatiques ( 8 sifflantes ou plus) se rencontrent assez fréquemment chez Euripide, Homère ou Aristophane, mais sont quasiment absents des œuvres conservées de Callimaque ou, chez les Latins, de Tibulle et Properce (pour ces deux derniers auteurs, P. A. Cronin ${ }^{26}$ considère un vers comme "polysigmatique » à partir du moment où l'on peut y relever la présence de 5 sifflantes). La prise en considération de la disposition des sifflantes proposée par T. Mommsen $^{27}$ à partir du vers 476 de la Médée d'Euripide parodié par Platon le Comique fournit également des résultats intéressants : le sigmatisme à proprement parler consiste, selon lui, en la succession d'une syllabe finale et d'une syllabe initiale commençant toutes deux par un sigma. De ce point de vue, les vers d'Euripide sont nettement plus sigmatiques que ceux des deux autres Tragiques et, là encore, les auteurs de la période hellénistique se montrent bien plus modérés. Le sigmatisme en général semble donc être une caractéristique de la langue grecque classique ${ }^{28}$ et avoir été évité par les Alexandrins et par les poètes latins alexandrinisants.

Dans le carmen 84 de Catulle, les vers 2, 7, 11 et 12 présentent une concentration de 5 ou 6 sifflantes et l'on peut relever deux cas de sigmatisme au sens de Mommsen : s(e) esse au v. 3 et esse sed au v. 12. Parmi les épigrammes (c. 69-116), cette pièce se distingue effectivement à la fois par le nombre de vers polysigmatiques et par le nombre de sigmatismes qu'elle comprend ${ }^{29}$. En outre, leur répartition confirme les observations de E. Vandiver puisque deux vers polysigmatiques se succèdent dans le dernier distique et que le dernier vers est, en sus, sigmatique au sens de Mommsen, avec une gradation par rapport au v. 3 où le sigmatisme était atténué par la présence de l'élision et n'était pas renforcé par la présence d'un $s$ final dans le mot précédent (se esse, 3 ; Ionios esse sed, 12).

AJPh, 30, 1909, p. 72-77, et sur l'ouvrage de T. Mommsen, Beiträge zu der Lehre von den griechischen Präpositionen, Berlin, 1895, p. 668-762.

${ }^{25}$ Voir Denys d'Halicarnasse (Comp. VI, 14, 20) qui décrit ce son comme déplaisant et en proscrit l'usage excessif et Quintilien (Inst. IX, 4, 37) qui recommande d'éviter les rencontres entre sifflante finale et sifflante initiale.

${ }^{26}$ P. A. Cronin, «Sigmatism in Tibullus and Propertius », CQ, 20, 1970, p. 174-180; voir p. 174.

${ }^{27}$ Mommsen, 1895, p. 669-672.

${ }^{28}$ Voir Michel, 1955, p. 140.

${ }^{29}$ Cette épigramme de 12 vers contient 2 sigmatismes au sens de Mommsen contre 6 pour le reste des épigrammes ( 295 vers), répartis dans des pièces différentes. Elle comprend 4 vers polysigmatiques (1 hexamètre à 5 sifflantes, 2 pentamètres à 5 sifflantes et 1 hexamètre à 6 sifflantes), concentration qui ne se retrouve que dans les pièces 103 et 115 (qui contiennent 3 vers polysigmatiques sur 4 et 8 vers respectivement). On relève, pour l'ensemble des épigrammes, 39 vers polysigmatiques répartis de la manière suivante : 5 sifflantes : 9 hexamètres et 16 pentamètres; 6 sifflantes : 5 hexamètres et 5 pentamètres ; 7 sifflantes : 3 pentamètres et 1 hexamètre. Ces poèmes ne présentent aucun vers qui contienne plus de 7 sifflantes. 
Il est donc probable que la question de la sifflante, qu'il s'agisse de sa prononciation en fin de mot ou de sa répétition, soit un domaine où s'est fait sentir l'influence du grec sur le latin. Dans ce domaine aussi, Arrius s'efforcerait d'apparaître comme un philhellène, mais pervertirait ce qui aurait pu être une marque de goût poétique et oratoire. La perversion ici consiste vraisemblablement en une prononciation trop marquée de la sifflante, combinée peutêtre avec des rencontres de consonnes disharmonieuses provoquées par la restauration de l's final $^{30}$ ou avec des allitérations ridicules.

\section{Tempêtes de neige, libations excessives et spirantisation}

Le troisième champ que nous explorerons concerne la question des hiatus, à laquelle nous amène une lecture de la fin du poème différente de celles qui ont été proposées jusqu'à présent. Mais il nous faut, au préalable, aborder ces lectures antérieures qui nous ont suggéré la recherche d'un jeu de mots final et expliquer en quoi, à nos yeux, elles ne sont pas satisfaisantes.

Le mouvement du carmen 84 semble indiquer que, après une pause marquant le soulagement aux v. 7-9, on se dirige, avec le cum inuersum et l'annonce du nuntius horribilis du v. 10, vers un climax, impression renforcée par ce que le genre épigrammatique suscite, en l'espèce, comme attente : le dernier vers ou, mieux, le dernier mot devrait constituer une pointe. Une partie de la critique considère que le retour inopiné de l'aspiration suffit à répondre à cette attente. À cela s'ajoute le fait que l'aspiration intempestive s'applique, au vers 12 , non plus à un mot latin ainsi hellénisé à tort, mais à un terme grec rencontré en territoire hellénophone : l'hellénisation, en Grèce même, d'un mot grec serait un comble ${ }^{31}$ !

Plusieurs chercheurs, cependant, ne jugent pas cette explication satisfaisante : elle ne leur paraît pas correspondre suffisamment à l'esthétique de la pointe. Certains ${ }^{32}$, pour pimenter la fin de la pièce, on proposé d'y lire un jeu de mots bilingue : Hionios représenterait

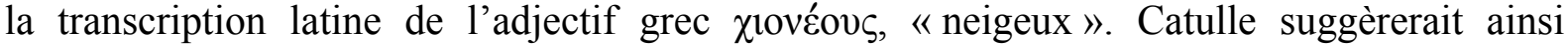
qu'Arrius est un piètre orateur, les mauvais littérateurs étant présentés, dans l'Antiquité, comme soufflant le froid. B. Einarson (1966) cite à l'appui de cette hypothèse les v. 136 sqq

\footnotetext{
${ }^{30}$ Voir la remarque de Quintilien à ce sujet (Inst. IX, 4, 37-38) : Ceterum consonantes quoque, earumque praecipue quae sunt asperiores, in commissura uerborum rixantur, ut s ultima cum $x$ proxima, quarum tristior etiam, si binae collidantur, stridor est, ut " ars studiorum ». Quae fuit causa et Seruio <Sulpicio>, ut dixi, subtrahendae s litterae quotiens ultima esset aliaque consonante susciperetur, quod reprehendit Luranius, Messala defendit. Nam neque Lucilium putat uti eadem ultima, cum dicit "Aeserninus fuit» et "dignus locoque», et Cicero in Oratore plures antiquorum tradit sic locutos. « Du reste, les consonnes aussi, celles surtout qui sont un peu dures, se heurtent dans la liaison des mots, par exemple l's final rencontrant l' $x$ initial du mot suivant; le sifflement est encore plus désagréable, lorsque ce sont deux $s$ qui entrent en contact, comme dans ars studiorum. C'est ce qui avait même entraîné Servius <Sulpicius>, comme je l'ai dit, à retrancher la lettre $s$ en finale, lorsque le mot suivant commençait par une autre consonne. Luranius l'en blâme, Messala le défend. Car il pense que Lucilius ne fait pas sentir l's final, lorsqu'il dit: Aeserninus fuit et dignus locoque, et Cicéron dans l'Orateur rapporte que beaucoup d'anciens ont adopté cette prononciation. » (trad. Cousin - André, 1978, CUF).

${ }^{31}$ J. Marouzeau, Traité de stylistique latine, Paris, 1946, p. 10, note que les aspirations fautives ont tendance à apparaître notamment dans les mots empruntés au grec : Orchus, Bosphorus, anchora.

${ }^{32}$ Harrison, 1915, B. Einarson, « On Catullus $84 », C P, 61,1966$, p. 187-188, Quinn, 1973, Della Corte, 1977, p. 346, Coraluppi, 1999.
} 
des Acharniens d'Aristophane dans lesquels des chutes de neige en Bulgarie sont mises en relation par l'un des personnages avec la représentation à Athènes, au même moment, d'une pièce du mauvais poète Théognis ; on pourrait aussi citer le poème 44 de Catulle dans lequel le poète se plaint d'avoir pris froid en lisant un discours de Sestius. Ce jeu de mots final serait préparé $^{33}$ par un certain nombre de termes qui appartiennent à la fois au vocabulaire technique linguistique et au vocabulaire technique météorologique : leniter et leuiter (8), horribilis (10), spiritus et asper.

Un autre chercheur (Nicholson, 1998) suggère, dans le même esprit, un jeu de mots différent : Hionios, qui est en relation de paronymie avec le nom grec du vin, oivvos, renverrait à l'adjectif Chios qui, substantivé, désigne le vin de Chios. Selon lui, le poème ne fustigerait pas un défaut de prononciation lié à l'inculture d'un parvenu qui veut faire le snob, mais viserait plutôt l'ivrognerie, dont les difficultés d'élocution d'Arrius ne seraient qu'une conséquence. J. Nicholson s'appuie, pour cette proposition de lecture, sur l'indication qu'Arrius ainsi que ses deux fils étaient des gourmets célèbres pour leurs excès (Hor., Sat. II, 3,86 et 243-246), sur le qualificatif de liber appliqué à l'oncle (5), qui renverrait non au statut d'affranchi ou d'homme libre, mais à l'un des noms de Bacchus ${ }^{34}$ - toute la famille serait composée d'ivrognes -, sur un jeu de mot entre poterat, du verbe posse, et la forme courte de plus-que-parfait du verbe potare équivalant à potauerat, sur les allitérations en [h] et en [s] et les répétitions de syllabes et de mots, caractéristiques selon lui de l'élocution d'un homme ivre, et enfin sur l'existence d'une variété de vin de Chios appelée « ariusienne » (Plin., H.N., XIV, 9, 73).

Plusieurs difficultés se présentent toutefois, concernant ces deux hypothèses.

La première est le manque de préparation du jeu de mots final. Dans le cas d'un jeu de

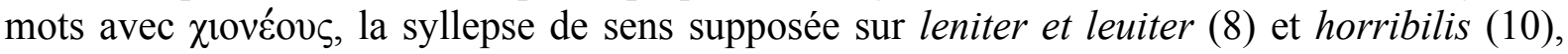
termes à la fois stylistiques et météorologiques, ne peut être perçue au moment où ces termes apparaissent dans le poème puisqu'il n'est pas question alors du climat; elle ne pourrait être activée qu'après coup, à partir du jeu de mots sur Hionios (12), qu'elle ne prépare donc pas. Quant aux deux autres termes invoqués, spiritus et asper, ils n'apparaissent tout simplement pas dans le poème, même s'ils sont suggérés dans la mesure où l'aspiration grecque est désignée en latin par l'expression spiritus asper. Dans le cas d'un jeu avec Xîos, semblablement, la syllepse de sens sur liber et le jeu sur la parononymie entre pŏterat et pōt(au)erat paraissent difficiles à percevoir en l'absence d'autres indices cotextuels concernant la boisson (dans le parallèle invoqué par J. Nicholson pour poterat, la présence des mots aqua et ebrius rend justement perceptible un tel jeu de mots : Mart. I, 26, 2, aqua totiens ebrius esse potes). Les allitérations en [h] et [s] et les répétitions ne sont pas non plus suffisamment caractéristiques en elles-mêmes de l'élocution d'un ivrogne pour préparer le jeu de mots final. D'une part, les répétitions de mots et de syllabes sont trop courantes chez

${ }^{33}$ D'après Coraluppi, 1999, qui s'appuie sur D. M. Jones, «Catulli nobile epigramma (Carm. LXXXIV) », PCA, 53, 1956, p. 25-26.

${ }^{34}$ L'idée que Liber du v. 5 serait un cognomen plutôt qu'un adjectif se trouve déjà chez Bell, 1915, D. N. Levin, "Arrius and His Uncle », Latomus 32, 1973, p. 587-594, et R. J. Baker et B. A. Marshall, "Auunculus liber (C. 84.5)», Mn. 30, 1977, p. 292-293. Toutefois, le sens d'« homme libre", suggérant que ce statut n'était pas toujours allé de soi dans la famille d'Arrius, s'inscrit bien dans le cadre de l'invective pratiquée par Catulle dans cette pièce et peut d'ailleurs constituer une exagération ; il ne nous semble donc pas nécessaire de l'écarter. 
Catulle pour pouvoir être invoquées $i^{35}{ }^{35}$; d'autre part, les exemples parallèles d'allitérations cités sont peu convaincants, et il faut garder à l'esprit le fait que l'interprétation d'une allitération peut être très variable ${ }^{36}$. Comme l'a d'ailleurs rappelé L. P. Wilkinson ${ }^{37}$, une allitération expressive ne fait que souligner le sens, elle ne l'exprime pas en elle-même : les allitérations ne peuvent donc pas constituer une preuve des imputations d'ivrognerie à l'encontre d'Arrius, elles peuvent seulement leur donner plus de relief si elles sont par ailleurs clairement présentes dans le poème. Enfin, il n'est pas non plus sûr que le contexte (ce que les lecteurs savaient de Quintus Arrius, puisque c'est sur cette identification du personnage que s'appuie J. Nicholson) permettait de faire de telles inférences: les passages invoqués d'Horace (Sat. II, 3, 86 et 243-246) présentent le personnage et ses fils comme des gourmets extravagants, mais pas comme des ivrognes (il n'y est d'ailleurs question que de plats exotiques et coûteux, sans mention de boissons).

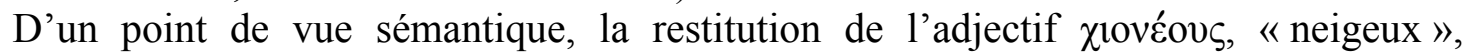
présente une autre difficulté. Si cette épithète évoque bien la froideur que dégagent les discours d'Arrius, la transformation de la mer ionienne en mer neigeuse ne constitue peut-être pas vraiment ce que l'on attendrait d'un nuntius horribilis. L. F. Coraluppi (1999) voudrait y lire une allusion à la tempête que dut affronter la flotte de Crassus après avoir quitté le port de Brindes pour son expédition en Syrie de 55 (expédition à laquelle on a tout lieu de croire que le départ d'Arrius en Syrie était lié). Mais il est obligé de passer pour cela par une association

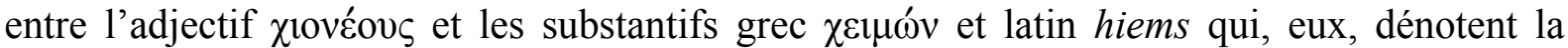
tempête, ce qui ne fait que compliquer encore un jeu de mots qui ne paraît déjà pas très facile à percevoir.

Mais la principale objection opposable à l'une et l'autre hypothèse est d'ordre phonétique, comme cela a du reste déjà été souligné ${ }^{38}$. Les deux propositions reposent en effet sur l'idée que le $h$ initial de Hionios serait l'équivalent d'un $\chi$ grec. On sait qu'à une date tardive le $\chi$ du grec a connu une prononciation spirantisée, qui est celle du grec moderne, et que le $h$ latin a dû avoir une prononciation similaire puisqu'on trouve des graphies tardives du type michi $\left(\mathrm{IV}^{\mathrm{e}} \mathrm{s}\right.$.). La date exacte à laquelle s'est produit ce changement phonétique en grec est difficile à déterminer. On considère habituellement qu'elle se situe au début $\mathrm{du}^{\mathrm{er}} \mathrm{s}$. de notre ère pour la labiale : le $\varphi$ grec a été rendu en latin par $p$ puis par ph (Pilipus, Philippus) et les premières transcriptions latines par $f$ datent du premier siècle avant notre ère mais ne deviennent fréquentes que deux siècles plus $\operatorname{tard}^{39} ; \mathrm{W}$. S. Allen ${ }^{40}$ cite à ce sujet l'anecdote (rapportée par Quintilien, Inst. I, 4, 14) selon laquelle Cicéron s'était moqué d'un témoin grec incapable de prononcer la première consonne du nom Fundanius ${ }^{41}$. Tout porte donc à croire que la consonne sourde aspirée labiale, dont l'existence de la spirante labiale sourde $f$ du latin permet de mieux évaluer l'évolution, commençait à peine à être spirantisée à l'époque de

\footnotetext{
${ }^{35}$ Sur la récurrence lexicale dans l'œuvre de Catulle, voir J. Évrard-Gillis, La Récurrence lexicale dans l'œuvre de Catulle. Étude stylistique, Liège, 1976, qui analyse la répétition ternaire de sic aux vers 5-6 de l'épigramme 84 comme un procédé d'emphase marquant une intention ironique (p. 205).

${ }^{36}$ On pourra se reporter par exemple à la longue liste des « harmonies imitatives » et « suggestives » auxquelles peuvent participer les allitérations en $s$ qui ont été étudiées par Michel, 1955 - valeurs parmi lesquelles ne figure toutefois pas celle que postule J. Nicholson.

37 "Onomatopoeia and the Sceptics », CQ, 36, 1942, p. 121-133; voir p. 121-122.

${ }^{38}$ Voir notamment Fordyce, 1961, p. 377.

${ }^{39}$ Biville, 1990, p. 67.

${ }^{40}$ W. S. Allen, Vox Graeca. A Guide to the Pronunciation of Classical Greek, Cambridge, $1987^{3}$ $\left(1968^{1}\right)$, p. 22-23.

${ }^{41}$ Voir aussi Biville, 1987, p. 30-31, qui indique que la transcription de $\chi$ par $h$ est rare est très tardive.
} 
Catulle. Les consonnes sourdes aspirées dorsale et dentale, quant à elles, n'ont été spirantisées que plus tardivement, en grec byzantin ${ }^{42}$. F. Biville conclut ainsi (1990, p. 202) : «Dans l'évolution des occlusives aspirées en spirantes, la palatale $\chi$ a été la plus résistante. Les manifestations de ce phénomène dans les emprunts sont très tardives (il s'agit essentiellement d'un phénomène médiéval). " Aussi est-il tout à fait hasardeux de s'appuyer sur une équivalence entre $h$ et $\chi^{43}$ L. F. Coraluppi (1999), conscient de ces faiblesses concernant

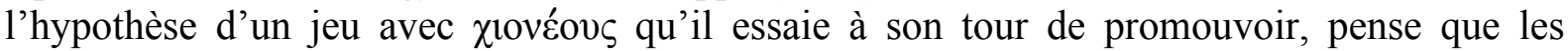
Romains, du moins le petit cercle de lettrés auquel s'adresse Catulle, percevaient l'équation entre leur $h$ initial et le $\chi$ initial du grec (tous deux issus d'une ancienne occlusive sonore aspirée $*^{h}{ }^{h}$-) dans des mots apparentés, comme par exemple hiems et $\chi 1 \omega ́ v$. En l'absence de témoignages antiques concernant de tels rapprochements (alors même que les grammairiens latins s'efforçaient autant que possible d'établir des équivalences entre leur langue et la langue grecque), l'hypothèse paraît aventureuse. ${ }^{44}$

En somme, le repérage des indices qui sont censés préparer la reconnaissance du jeu de mots final suppose déjà la connaissance de ce jeu de mots et la proximité phonique entre Hionios et $\chi$ lovéov̧ ou Xîos n'est sans doute qu'une chimère. Ces deux propositions de lecture nous semblent donc devoir être écartées.

\section{Cacophonie des hiatus}

À titre d'expérience, nous suivrons cependant la piste que ces deux lectures ont empruntée en cherchant un jeu de mots dans la forme Hionios. Pour éviter les ornières que constituent la difficulté d'un jeu de mots bilingue et le rapprochement du $h$ latin et du $\chi$ grec, nous bornerons nos spéculations à la langue latine, ce qui nous amène à nous demander si le début du mot Hìonios, avec aspiration, ne serait pas susceptible d'évoquer le radical latin $h \bar{l}-$. Ce radical apparaît sous cette forme dans $h \bar{l}$-sc-ere et le préverbé de-hi-sc-ere, et sous sa variante avec voyelle abrégée devant une autre voyelle dans hi-āre, hi-a-tus, hi-et-āre, hi-ul$c$-ãre et hi-ul-cus ${ }^{45}$. Seuls les deux premiers phonèmes de Hionios joueraient dans cette identification du radical $h \bar{l}$-, mais les différentes valeurs sémantiques de ce radical et de la famille de mots qui s'y rattache semblent de nature à assurer cette reconnaissance. En effet, à

\footnotetext{
${ }^{42}$ Voir Biville, 1990, p. 72. Cette observation est en accord avec ce que laissent supposer les données typologiques: celles-ci semblent indiquer une propension à la spirantisation plus grande pour la labiale que pour la dorsale et la dentale. C'est ce que l'on peut observer en ossète, en hongrois, en arménien, en celtique et à l'initiale en allemand (A. Christol, communication personnelle). Voir aussi D. Seigneur Froli, De la lénition des «codas» initiales en grec. Étude diachronique de la spirantisation grecque dans un cadre $C V C V$, Mémoire de DEA de l'Université de Nice-SophiaAntipolis, sous la direction de T. Scheer, 2001.

${ }^{43}$ Du reste, si le $h$ de hinsidias, aux vers 2 et 4, équivaut à l'esprit rude du grec, comme cela est généralement admis, on ne voit pas pourquoi celui de Hionios représenterait un $\chi$. Une spirante aurait en outre dû faire position dans le vers, allongeant le sed qui précède, qu'elle soit notée $h$ ou $c h$.

${ }^{44}$ Qui, aujourd'hui, même parmi les personnes cultivées, s'est déjà fait consciemment la réflexion que le $p$ - initial du français correspond, dans un certain nombre de mots hérités, au $f$-initial de l'anglais (par exemple, père - father à côté de mère - mother) et serait susceptible d'en déduire une équivalence du même type (la correspondance, par exemple, entre pied et foot), qui plus est dans le cadre allusif d'un jeu de mots?

${ }^{45}$ L'existence, en latin, de ces différentes formes de sens proche assure l'identification par les locuteurs d'un radical commun $h \bar{l}$ - (et non hīa- par exemple).
} 
un premier niveau, l'idée de flots soudain béants laissant découvrir la profondeur du gouffre marin paraît répondre de manière satisfaisante à l'attente créée par le nuntius horribilis du vers $10^{46}$. Les verbes hiare et dehiscere, qui dénotent le plus souvent l'ouverture d'une fissure terrestre, se trouvent aussi employés pour désigner celle d'un abîme maritime. Ainsi chez Virgile, dans la description de la tempête du chant I de l'Énéide (v. 106-107) : hi summo in fluctu pendent, his unda dehiscens / terram inter fluctus aperit, « les uns sont suspendus à la crête du flot, à d'autres l'onde béante découvre la terre entre les flots " (trad. Perret - Le Bonniec, 1977, CUF), ou dans la Pharsale de Lucain (V, 640-641) lors du récit de la tentative faite par César pour traverser la mer Adriatique pendant une nuit de tempête sur une petite embarcation : cumque tumentes / rursus hiant undae, uix eminet aequore malus, « et quand les ondes s'entr'ouvrent en bouillonnant, à peine le mât fait-il saillie à la surface » (trad. Bourgery - Ponchont, 1926, CUF modifiée) ${ }^{47}$.

À un second niveau, dans le contexte de ce poème portant sur des aspects de l'elocutio, le radical $h \bar{l}$ - peut faire penser au sens de "parler », "déclamer », " chanter » pris par hiare et hisco (en passant par «ouvrir la bouche pour parler» ou « faire entendre par la bouche ouverte $»)^{48}$ : l'Oxford Latin Dictionary indique «to utter with extravagant articulation » en s'appuyant sur Properce (II, 31,6) et Perse $(5,3)$. Dans cette épigramme qui traite de prononciation, qui plus est de la prononciation avec une articulation exagérée (quantum poterat, 4) d'un personnage qui avait peut-être la prétention d'être un orateur (si l'identification de Quintus Arrius est valide), et où les verbes de parole sont omniprésents, finir sur une nouvelle évocation d'un uerbum dicendi ne serait pas déplacé. Mais ce qui nous intéresse au premier chef dans cette hypothèse, c'est la possibilité d'une évocation, encore plus spécifiquement liée au contexte des débats sur l'elocutio, du sens technique pris par hiare, hiatus, hiulcare et hiulcus chez les orateurs et les grammairiens qui traitent de la question de l'hiatus.

Cette question d'elocutio est évoquée par Cicéron peu avant celle du $h$ et du $s:$ les rencontres de voyelles sont à éviter ${ }^{49}$. Mais est-ce à dire qu'il faut, en cas de rencontre, pratiquer l'élision (qu'elle aboutisse à la disparition complète de la voyelle finale ou à sa prononciation rapide devant la voyelle initiale en une même émission de voix ${ }^{50}$ ), ou plutôt qu'il faut placer les mots de sorte à éviter complètement ces rencontres de voyelles ? On retient généralement la première interprétation, étant donné que les discours de Cicéron même les plus soignés contiennent un bon nombre de rencontres entre une voyelle finale et une voyelle initiale et que l'alternative au hiatus présentée en Orat. 77 est l'action désignée par le

\footnotetext{
${ }^{46}$ L'idée que le nuntius horribilis renvoie à une tempête, voire plus précisément à celle qu'essuya Crassus à son départ de Brindes pour la province de Syrie, se trouve chez les tenants de l'hypothèse

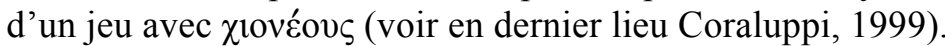

${ }^{47}$ Voir aussi Sénèque, $A g .499$ (récit de la tempête qui dispersa la flotte grecque au retour de Troie), Valerius Flaccus I, 624 (description de la tempête essuyée par les Argonautes au début de leur voyage) et VIII, 329 (tempête soulevée par Junon contre les Colques) ou encore Val. Fl. II, 587 et Stat. Silv. III, 2, 63 .

${ }^{48}$ Sens attesté chez Cicéron, Tite-Live et les poètes augustéens, Properce et Ovide notamment.

${ }^{49}$ Cic., Orat. 150 : ne extremorum uerborum cum insequentibus primis concursus aut hiulcas uoces efficiat aut asperas, "de façon que la rencontre des fins de mots avec le début des suivants ne produise ni hiatus ni cacophonie » (trad. Yon, 1964, CUF). On trouve une recommandation similaire dans la Rhétorique à Hérennius (IV, 12, 18).

${ }^{50}$ Voir à ce propos J. Soubiran, L'Élision dans la poésie latine, Paris, 1966, passim.
} 
verbe coagmentare ${ }^{51}$; c'est ce que semble indiquer également l'enchaînement, au paragraphe 153 de l'Orateur, entre la question de la prononciation des voyelles en hiatus et celle de la prononciation de la sifflante finale: dans les deux cas, une prononciation "contractée », illustrée pour la sifflante par la graphie multi' modis, était le plus souvent adoptée (Sed quid ego uocalis? Sine uocalibus saepe breuitatis causa contrahebant, "Mais que parlé-je de voyelles? Sans voyelles souvent, on faisait des contractions, pour raccourcir », trad. Yon, 1964, CUF). Il était donc recommandé à l'orateur d'éviter, comme le font les poètes (indicant omnes poetae, Orat. 152), la prononciation en hiatus. Cicéron souligne sur ce point la différence entre l'usage latin et l'usage grec, en $\operatorname{prose}^{52}$ comme en poésie $^{53}$. En ce qui concerne la prose, l'usage semble avoir varié d'un auteur grec à l'autre : si Isocrate évitait l'hiatus au point de ne pas placer l'un à la suite de l'autre un mot se terminant par une voyelle et un mot à initiale vocalique, la plupart des autres orateurs, à côté d'un certain nombre d'élisions dont la notation a été conservée, pratiquaient occasionnellement l'hiatus. Ce dernier permettait de marquer une légère pause et de mettre ainsi certains termes en relief ou de rendre le ton plus solennel ${ }^{54}$. C'est de cet usage sans doute que se réclamaient les orateurs néo-attiques dont le style simple, affectant une composition relâchée, présentait des hiatus que Cicéron ne jugeait pas dépourvus de charme ${ }^{55}$. On retrouverait donc ici, si l'on admettait cette hypothèse concernant la lecture de Hīonios, une faute de goût qui consisterait en un usage sans doute excessif d'un trait de prononciation mis au goût du jour par les orateurs hellénisants.

Chez les poètes latins, le maintien en hiatus de la finale d'un mot de plus d'une syllabe n'est normalement pas admis ; quand l'hiatus est employé, c'est généralement par imitation de l'usage homérique : ainsi Virgile ne l'admet-il que dans des vers contenant des mots grecs ou des hellénismes de versification ${ }^{56}$. Mais l'usage a connu des variations : les poètes les plus anciens, Livius Andronicus et Naevius, ne l'excluaient pas systématiquement et c'est Ennius qui en a très nettement restreint l'emploi (c'est ce que nous apprend Cicéron, Orat. 152, et ce que confirment les recherches faites par J. Soubiran ${ }^{57}$ ). Si l'on consulte les relevés de A. Siedow $^{58}$, on s'aperçoit toutefois que l'hiatus a été évité avec encore plus de soin par les auteurs qui lui ont succédé, Lucilius, Cicéron et Lucrèce, chez lesquels le pourcentage

\footnotetext{
${ }^{51}$ Voir L. Laurand, Études sur le style des discours de Cicéron, Paris, 1925-1927, p. 125-126, et A. M. Riggsby, «Elision and Hiatus in Latin Prose », ClAnt, 10, 1991, p. 328-343, en particulier p. 330.

52 Orat. 152 : Sed Graeci uiderint; nobis ne si cupiamus quidem distrahere uoces conceditur, « Mais ceci regarde les Grecs; nous, même si nous le voulions, nous n'avons pas le droit de séparer les voyelles » (trad. Yon, 1964, CUF).

${ }^{53}$ Orat. 153, pour commenter les citations de Naevius et d'Ennius: Hoc idem nostri saepius non tulissent, quod Graeci laudare etiam solent, «Nos compatriotes n'auraient pas toléré trop souvent cette licence que les Grecs ont l'habitude même de louer » (trad. Yon, 1964, CUF).

${ }^{54}$ Voir L. Pearson, « Hiatus and Its Purposes in Attic Oratory », AJPh, 96, 1975, p. 138-159.

55 Orat. 77: Verba etiam uerbis quasi coagmentare neglegat. Habet enim ille tamquam hiatus concursu uocalium molle quiddam et quod indicet non ingratam neglegentiam de re hominis magis quam de uerbis laborantis. "Qu'il néglige même d'agencer en quelque sorte les mots aux mots. Car l'espèce d'hiatus résultant de la rencontre des voyelles a quelque chose de souple et qui indique la négligence qui ne déplaît point d'un homme préoccupé des choses plus que des mots. » (trad. Yon, 1964, CUF).

${ }^{56}$ L. Nougaret, Traité de métrique latine classique, Paris, 1948, p. 51-52.

${ }^{57}$ Soubiran, 1966, p. 43-44 et 52-53.

${ }^{58}$ De elisionis, aphaeresis, hiatus usu in hexametris Latinis ab Ennio usque ad Ovidii tempora, Greifswald, 1911, p. 59.
} 
d'hiatus dans les hexamètres oscille entre 0,1 et $0,2 \%$ (il est de 1, $1 \%$ chez Ennius). Or, ce pourcentage s'accroît dans les hexamètres catulliens $(0,8 \%)^{59}$, tendance confirmée par les chiffres virgiliens $(0,5 \%)$ : il est permis de penser que l'usage de l'hiatus a été, dans une certaine mesure, remis au goût du jour par les poètes alexandrinisants ${ }^{60}$. L'hiatus, jamais complètement banni de la poésie latine, était apprécié dans la mesure où il pouvait produire des rencontres vocaliques étrangères à la langue latine et l'hiatus interne présenté par nombre de mots grecs n'était pas le moindre de leurs attraits pour les poètes latins, qui y avaient souvent recours ${ }^{61}$. Ionios, avec son premier $i$ long, comporte précisément l'un de ces hiatus internes propres aux termes grecs ; on peut se demander si cet hiatus, qui suit immédiatement, dans sa dernière occurrence (Hīonios, v. 12), le $h \bar{l}$ - initial, ne serait pas de nature à permettre la reconnaissance d'un jeu de mots avec le radical signifiant « être en hiatus / produire un hiatus ».

Pour la question de l'hiatus comme pour celles de l'aspiration et de la sifflante, il y aurait donc concordance entre l'usage oratoire et l'usage poétique de la fin de l'époque républicaine, tous deux influencés par la pratique des auteurs grecs. Quelques hiatus, employés à bon escient, dans un discours ou un poème, peuvent charmer l'oreille, mais un usage excessif reste une marque de rusticité.

\section{Conclusion : Hellénomanie et débats stylistiques chez les orateurs et les poètes de la fin de la République}

Cette exploration de l'épigramme 84 de Catulle nous a conduite à des conclusions destinées à demeurer conjecturales sur certains points : rien, sans doute, ne nous permettra jamais de nous assurer que Catulle entendait bien achever cette pièce sur un jeu de mots ni, si tel était le cas, de savoir de façon certaine quel était le terme que devait évoquer la forme Hionios. Si nous nous sommes permis, après d'autres, d'avancer une hypothèse à ce sujet, c'est avant tout pour la valeur heuristique de cette démarche. Elle nous a amenée, en effet, à aborder la question de l'hiatus dans la poésie et la prose latines. Ce sont ainsi trois aspects de l'elocutio, la prononciation de l'aspiration et des consonnes aspirées, celle de la sifflante

\footnotetext{
${ }^{59}$ La question de savoir s'il faut conserver ou non le texte des poèmes catulliens tel qu'il nous a été transmis dans les quelques passages où il présente des hiatus a été au centre de nombreux débats. Il ne nous semble pas, contrairement à ce que proposent G. P. Goold, « A New Text of Catullus », Phoenix, 12, 1958, p. 106-111, ou J. M. Trappes-Lomax, "Catullus 107: Removing the Hiatus and Other Textual Suggestions ", Phoenix, 55, 2001, p. 304-312 et 2007, p. 9-10, que les vers contenant des hiatus doivent être systématiquement ou presque systématiquement corrigés, d'autant plus que la plupart de ces hiatus sont inspirés de pratiques grecques, comme l'ont montré M. Zicàri, "Some Metrical and Prosodical Features of Catullus' Poetry », Phoenix, 18, 1964, p. 193-205, et D. T. Benediktson, « Horribiles ultimosque Britannos », Glotta, 68, 1990, p. 120-123.

${ }^{60}$ Voir en ce sens les remarques de W. S. Allen, Accent and Rythm. Prosodic Features of Latin and Greek: A Study in Theory and Reconstruction, Cambridge, 1973, p. 143, qui note que l'emploi d'hiatus correspond presque toujours à une imitation du grec dans la poésie latine classique alors que tel n'était pas le cas dans la poésie latine archaïque.

${ }^{61}$ Voir à ce propos N. I. Herescu, La Poésie latine. Étude des structures phoniques, Paris, 1960, p. 5967, et Coleman, 1999, p. 46.
} 
finale et celle des voyelles en hiatus, traités tous trois dans la même section de 1'Orateur ${ }^{62}$, que nous avons pu étudier à travers cette épigramme. Il s'avère que ces trois questions de prononciation sont, d'une part, débattues par les orateurs de la fin de la République et qu'elles constituent, d'autre part, des enjeux stylistiques importants pour les poètes hellénisants de l'époque, comme Catulle. Orateurs et poètes subissent en effet l'influence grecque et sont, par ailleurs, confrontés à l'évolution de la prononciation dans la langue parlée de l'élite romaine, laquelle est également influencée par le modèle grec. Restauration de l'aspiration initiale, introduction des consonnes aspirées, restauration de la sifflante finale, choix d'une prononciation en hiatus dans certains cas de rencontres de voyelles: dans quelle mesure discours et poèmes doivent-ils refléter ces changements? Autant de questions qui se posaient inévitablement à ceux qui se mêlaient d'écrire et auxquelles un Catulle et un Cicéron paraissent avoir apporté, chacun dans son domaine, et avec les formes d'expression qui leur sont propres, des réponses similaires, enregistrant ces évolutions du goût contemporain et reconnaissant l'attrait du modèle grec tout en conservant une certaine retenue.

C'est cette retenue, précisément, ainsi que le discernement et la distinction qu'elle suppose, qui font cruellement défaut à Arrius. Comme Asinius, stigmatisé dans un autre poème, Arrius est pris pour cible par Catulle parce qu'il prétend appartenir à une certaine élite urbaine et lettrée sans posséder les qualités requises : il cède sans retenue à l'hellénomanie ambiante et ses discours ne sont qu'une caricature de la production littéraire raffinée des auteurs hellénisants dont Catulle fait partie.

\author{
Frédérique FLECK \\ École normale supérieure (Paris)
}

\footnotetext{
${ }^{62}$ Orat. $150-153$ pour l'hiatus, 153 et 161 pour la sifflante, 160 pour l'aspiration ; il s'agit du passage où, à propos de la collocatio uerborum, Cicéron traite de la compositio en s'arrêtant sur divers points qui relèvent plus, en fait, de la correction (latinitas) que de l'ornementation.
} 